\title{
Autoimmune Encephalitis: An Unusual Presentation as Nonconvulsive Status Epilepticus
}

\author{
Deepak Solanki' Saurabh Anand ${ }^{1}$ \\ ${ }^{1}$ Department of Neuroanesthesia and Neurocritical Care, Artemis
Hospital, Gurugram, Haryana, India
}

\begin{abstract}
Address for correspondence Deepak Solanki, MBBS, MD Anaesthesiology, Department of Neuroanesthesia and Neurocritical Care, Artemis Hospital, Sector 51, Gurugram 122001, Haryana, India (e-mail: dr.dsolanki@gmail.com).
\end{abstract}

\begin{abstract}
Keywords

- autoimmune encephalitis

- cerebrospinal fluid

- electroencephalogram

- nonconvulsive status epilepticus

When refractory status epilepticus (RSE) occurs in an individual without any history of epilepsy and no immediate underlying etiology is found, it is referred to as new-onset refractory status epilepticus (NORSE). This clinical scenario may be notoriously difficult to treat and does not respond to initial medications. In cases of NORSE in which an etiology is found, antibody-mediated disorders are the most common cause. Autoimmune encephalitis refers to a diverse group of neuropsychiatric disorders and can present with an array of symptoms many of which make diagnosis difficult due to similarities in clinical, imaging, and laboratory findings with respect to other forms of autoimmune or infectious encephalitis. This case report highlights how a patient with acute-onset history, showing generalized periodic epileptiform discharges on electroencephalogram (EEG) but with no prior history of seizures, was eventually diagnosed as autoimmune encephalitis based on clinical findings, cerebrospinal fluid (CSF) reports, and EEG analysis.
\end{abstract}

\section{Introduction}

Autoimmune encephalitis as an entity can have a variable pattern of clinical presentation. There have been a few case reports pertaining to "nonconvulsive status epilepticus" (NCSE) presentation of autoimmune encephalitis, especially anti- $N$-methyl-d-aspartate (NMDA) receptor encephalitis. ${ }^{1,2}$

We present a case in which an unusual presentation of NCSE was diagnosed and treated successfully as autoimmune encephalitis after due exclusion of other possible diagnoses by correlating clinical findings, cerebrospinal fluid (CSF) reports, and electroencephalogram (EEG) analysis of the patient.

\section{Case History}

A 58-year-old woman presented to the intensive care unit (ICU) with chief complaints of history of acute behavioral change since 3 days in the form of confusional episodes, forgetfulness, drowsiness, dizziness, and episodic disorientation to time and place. The patient had prior history of carcinoma head of pancreas, post-Whipple's surgery, post-chemotherapy, with hepatic metastatic disease on positron emission tomography-computed tomography (PET-CT) scan (with no metastases in the brain).

Glasgow coma scale (GCS) score on arrival in ICU was E4V4M6. Pupils were $2 \mathrm{~mm}$ bilaterally reacting to light. Power was $2 / 5$ in all four limbs. There was no history of loss of consciousness, vomiting, convulsions, or neck rigidity. Vitals were stable at presentation. Magnetic resonance imaging (MRI) of the brain with contrast showed non-specific white matter ischemic changes. EEG showed generalized periodic epileptiform discharges (suggestive of NCSE) ( - Fig. 1).

Injections midazolam $2 \mathrm{mg}$ intravenously (stat), Levipil $1.5 \mathrm{~g}$, and Lacoset $200 \mathrm{mg}$ intravenously were loaded, and maintenance doses were charted. Baseline investigations were all normal.

The patient was given injection midazolam, but repeat EEG still showed epileptiform discharges. Hence, decision was taken to put the patient on midazolam infusion to achieve burst suppression. Thus, she was electively intubated for airway protection and for achieving burst suppression using midazolam. Midazolam infusion was initially started at $0.2 \mathrm{mg} / \mathrm{kg} / \mathrm{h}$, which gradually increased to $0.5 \mathrm{mg} / \mathrm{kg} / \mathrm{h}$ to
DOI https://doi.org/ $10.1055 / \mathrm{s}-0039-1685246$ ISSN 2348-0548.
Copyright @2020 Indian Society of Neuroanaesthesiology and Critical Care
License terms

() (1) $\Theta \circledast$ 
achieve burst suppression. On achieving burst suppression, continuous EEG monitoring (for 36 hours) was done to document the same (-Fig. 2).

With a provisional diagnosis of autoimmune encephalitis in mind, the patient was empirically given injection methylprednisolone $1 \mathrm{~g}$ OD (for 5 days).

During this period, diagnostic lumbar puncture was also performed. CSF cytology and biochemistry were normal. CSF biofire meningitis panel, autoimmune panel, and paraneoplastic panel were all negative.

Midazolam was then gradually tapered off over 36 hours and stopped. EEG done after stoppage of midazolam showed no epileptiform discharges (-Fig. 3).

The patient was gradually weaned off the ventilatory support and extubated.

The patient's EEG prior to discharge from ICU showed diffuse encephalopathy but no epileptiform discharges (-Fig. 4).

\section{Discussion}

Nonconvulsive status epilepticus accounts for $25 \%$ of all cases of status epilepticus. Criteria for diagnosis of NCSE are ${ }^{3,4}$

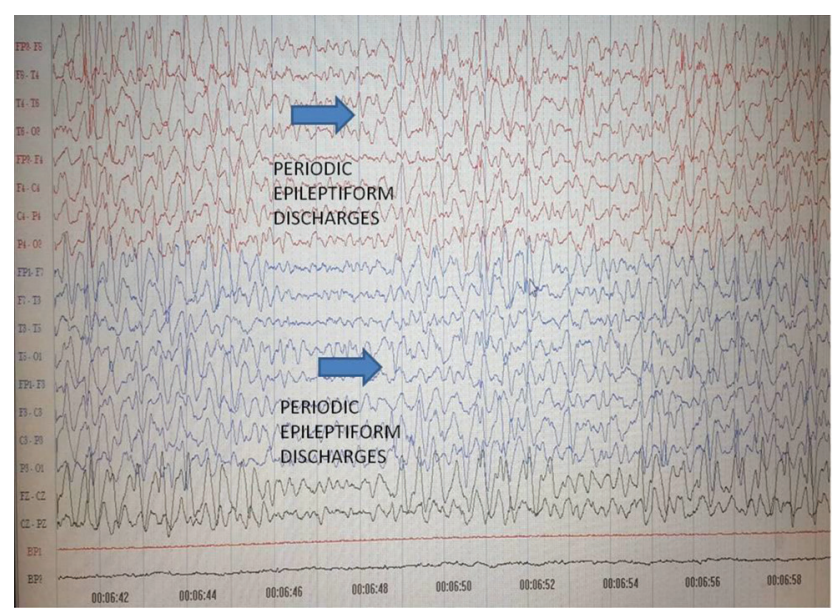

Fig. 1 EEG showing generalized periodic epileptiform discharges on admission. EEG, electroencephalogram.

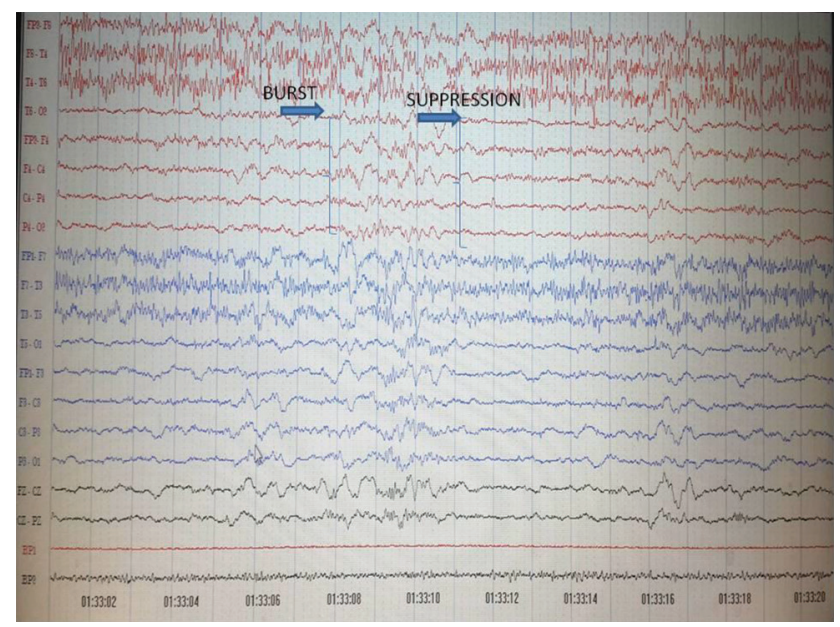

Fig. 2 EEG showing burst suppression pattern on midazolam infusion. EEG, electroencephalogram.
Altered consciousness with any of the following EEG changes noted for $\geq 5$ minutes:

- Repetitive focal or generalized epileptiform discharges (spikes, poly-spikes, sharp waves, sharp- and slow-wave complexes) at $\geq 3 \mathrm{~Hz}$.

- Sequential, rhythmic, periodic, or quasi-periodic discharges at $\geq 1 \mathrm{~Hz}$ and unequivocal evolution in frequency, morphology, or location.

- Repetitive focal or generalized epileptiform discharges at $\leq 3 \mathrm{~Hz}$ with significant clinical improvement or appearance of previously absent normal EEG background following administration of a rapidly acting antiepileptic drug (AED).

Refractory status epilepticus (RSE) is defined as continued clinical/electrographic seizures after adequate dosing of initial benzodiazepine, followed by second-line AED. It is associated with worse prognosis than non-RSE.

Our case was a unique case of difficult diagnosis of NCSE as the patient had symptoms of only confusion and disorientation. Strong suspicion was the key.

In our case, RSE occurred in a patient who never had a history of epilepsy and no immediate underlying etiology.

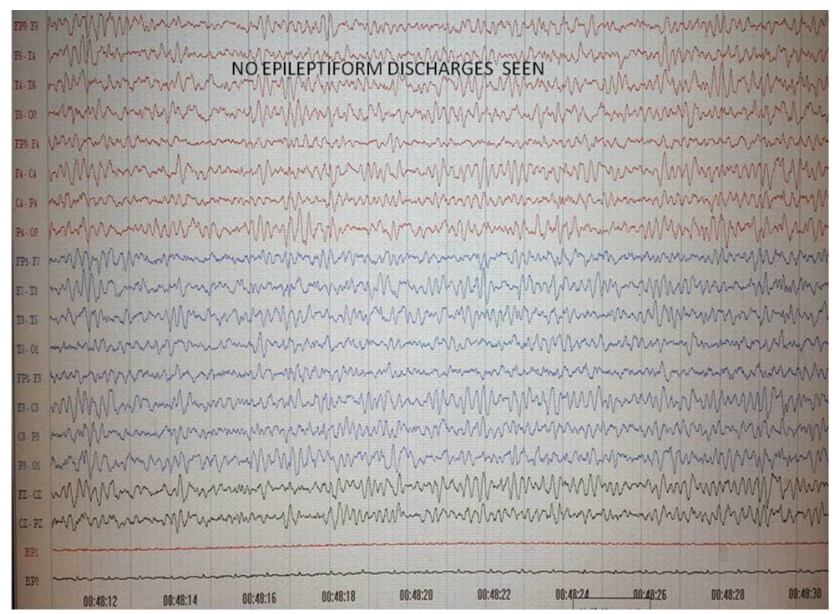

Fig. 3 EEG showing no epileptiform discharges (post-stoppage of midazolam). EEG, electroencephalogram.

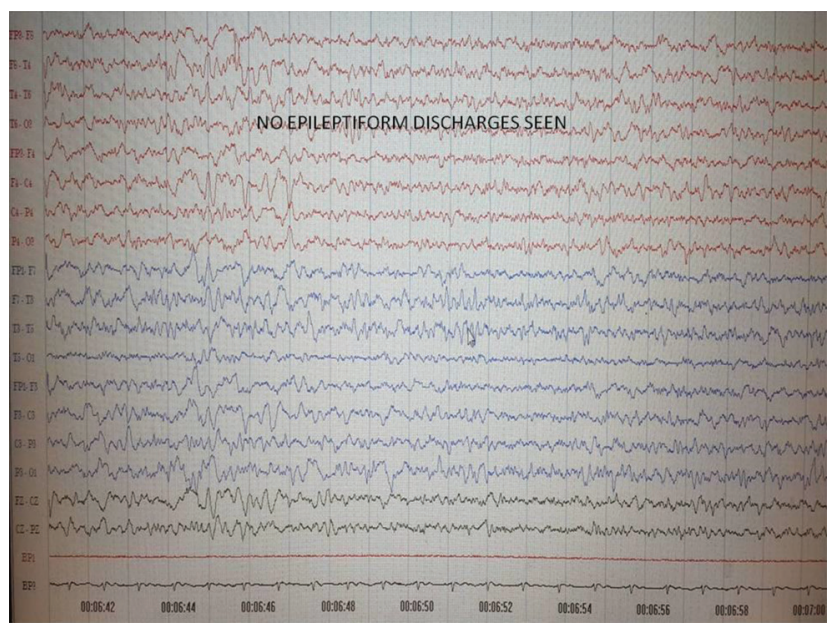

Fig. 4 EEG showing diffuse encephalopathy but no epileptiform discharges. EEG, electroencephalogram. 
These cases fall under ambit of new-onset refractory status epilepticus (NORSE). ${ }^{5-7}$ Commonly identified etiologies under NORSE include autoimmune encephalitis (19\%) and paraneoplastic encephalitis (18\%), and hence suspicion for autoimmune encephalitis versus paraneoplastic encephalitis was made initially. ${ }^{7}$

Owing to NCSE, continuous EEG monitoring (over 36 hours) was done to record burst suppression and subsequent response to AED therapy (cessation of seizures), before tapering of midazolam was initiated. Expert consensus dictates increasing anesthesia to achieve an electrographic burst suppression pattern, characterized by 2 to 10 second of background suppression $(<5 \mathrm{mV})$ with interspersed 1 to 2 seconds bursts of cerebral activity. ${ }^{8}$ Considering the prior history of carcinoma pancreas, post-Whipple's surgery, post-chemotherapy, and metastatic liver disease (immunosuppressed) status, burst (3-4 seconds) followed by suppression (5 seconds) were considered acceptable in this case. On the lines of suspected autoimmune encephalitis, injection methylprednisolone course of $1 \mathrm{~g}$ OD for 5 days was given, and the patient responded to it. Had the patient not responded to immunosuppression, intravenous immunoglobulins or plasmapheresis would have been the next line of treatment., 10

Despite CSF autoimmune panel reports coming "negative," this unusual case of NCSE presentation was considered to be autoimmune-mediated because

- Autoimmune encephalitis is the most common etiology for NORSE, and negative antibody tests do not rule out immune-mediated process as many autoantibodies are yet to be characterized.

- The patient responded to a 5-day course of intravenous methylprednisolone.

- Paraneoplastic encephalitis was ruled out in this case as CSF paraneoplastic panel report came "negative," and PET-CT scan had ruled out any metastasis in the brain.

This unusual case of autoimmune encephalitis manifesting as NCSE was diagnosed based on clinical symptoms, serial EEGs, and CSF analysis. The patient was successfully treated with AEDs (levetiracetam, lacosamide, and midazolam) and intravenous steroids (methylprednisolone) and was discharged home in a stable condition.

\section{Conclusion}

As a presentation of autoimmune encephalitis, NCSE requires high level of suspicion. Early EEGs with prompt and aggressive treatment can improve patient outcomes drastically.

\section{Conflict of Interest}

None declared.

\section{References}

1 Kim H, Ryu H, Kang JK. Anti-NMDA receptor antibody encephalitis presenting with unilateral non-convulsive status epilepticus in a male patient. J Epilepsy Res 2015;5(1):17-19

2 Goldberg EM, Taub KS, Kessler SK, Abend NS. AntiNMDA receptor encephalitis presenting with focal non-convulsive status epilepticus in a child. Neuropediatrics 2011;42(5):188-190

3 Young GB, Jordan KG, Doig GS. An assessment of nonconvulsive seizures in the intensive care unit using continuous EEG monitoring: an investigation of variables associated with mortality. Neurology 1996;47(1):83-89

4 Chong DJ, Hirsch LJ. Which EEG patterns warrant treatment in the critically ill? Reviewing the evidence for treatment of periodic epileptiform discharges and related patterns. J Clin Neurophysiol 2005;22(2):79-91

5 Wilder-Smith EP, Lim EC, Teoh HL, et al. The NORSE (new-onset refractory status epilepticus) syndrome: defining a disease entity. Ann Acad Med Singapore 2005;34(7):417-420

6 Costello DJ, Kilbride RD, Cole AJ. Cryptogenic new onset refractory status epilepticus (NORSE) in adults-Infectious or not? J Neurol Sci 2009;277(1-2):26-31

7 Gaspard N, Foreman BP, Alvarez V, et al; Critical Care EEG Monitoring Research Consortium (CCEMRC). New-onset refractory status epilepticus: Etiology, clinical features, and outcome. Neurology 2015;85(18):1604-1613

8 Van Ness PC. Pentobarbital and EEG burst suppression in treatment of status epilepticus refractory to benzodiazepines and phenytoin. Epilepsia 1990;31(1):61-67

9 Toledano M, Britton JW, McKeon A, et al. Utility of an immunotherapy trial in evaluating patients with presumed autoimmune epilepsy. Neurology 2014;82(18):1578-1586

10 Khawaja AM, DeWolfe JL, Miller DW, Szaflarski JP. New-onset refractory status epilepticus (NORSE)-the potential role for immunotherapy. Epilepsy Behav 2015;47:17-23 